\title{
A narrative review of electrical impedance tomography in lung diseases with flow limitation and hyperinflation: methodologies and applications
}

\author{
Ling Sang ${ }^{1 \#}$, Zhanqi Zhao ${ }^{2,3 \#}$, Zhimin Lin ${ }^{1 \#}$, Xiaoqing Liu ${ }^{1}$, Nanshan Zhong ${ }^{1}$, Yimin Li $^{1}$ \\ ${ }^{1}$ State Key Laboratory of Respiratory Diseases, Guangzhou Institute of Respiratory Health, Guangzhou Medical University, the First Affiliated \\ Hospital of Guangzhou Medical University, Department of Crit Care Med, Guangzhou, China; ${ }^{2}$ Department of Biomedical Engineering, Fourth \\ Military Medical University, Xi'an, China; ${ }^{3}$ Institute of Technical Medicine, Furtwangen University, Villingen-Schwenningen, Germany \\ Contributions: (I) Conception and design: X Liu, N Zhong, Y Li; (II) Administrative support: Z Lin; (III) Provision of study materials or patients: L \\ Sang, Z Zhao; (IV) Collection and assembly of data: L Sang, Z Zhao; (V) Data analysis and interpretation: L Sang, Z Zhao; (VI) Manuscript writing: \\ All authors; (VII) Final approval of manuscript: All authors. \\ \#These authors contributed equally to this work. \\ Correspondence to: Dr. Nanshan Zhong; Dr. Yimin Li. State Key Laboratory of Respiratory Diseases, Guangzhou Institute of Respiratory Health, \\ the First Affiliated Hospital of Guangzhou Medical University, Department of Crit Care Med, Guangzhou, China. Email: nanshan@vip.163.com; \\ dryiminli@vip.163.com.
}

\begin{abstract}
Electrical impedance tomography (EIT) is a functional radiation-free imaging technique that measures regional lung ventilation distribution by calculating the impedance changes in the corresponding regions. The aim of the present review was to summarize the current literature concerning the methodologies and applications of EIT in lung diseases with flow limitation and hyperinflation. PubMed was searched up to May 2020 to identify studies investigating the use of EIT in patients with asthma, bronchiectasis, bronchitis, bronchiolitis, chronic obstructive pulmonary disease, and cystic fibrosis. The extracted data included study design, EIT methodologies, interventions, validation and comparators, population characteristics, and key findings. Of the 44 included studies, seven were related to simulation, animal experimentation, or reconstruction algorithm development with evaluation on patients; 27 studies had the primary objective of validating EIT technique and measures including regional ventilation distribution, regional EITspirometry parameters, end-expiratory lung impedance, and regional time constants; and 10 studies had the primary objective of applying EIT to monitor the response to therapeutic interventions, including various ventilation supports, patient repositioning, and airway suctioning. In pediatric and adult patients, EIT has been successfully validated for assessing spatial and temporal ventilation distribution, measuring changes in lung volume and flow, and studying regional respiratory mechanics. EIT has also demonstrated potential as an alternative or supplement to well-established measurement modalities (e.g., conventional pulmonary function testing) to monitor the progression of obstructive lung diseases, although the existing literature lacks prediction values as references and lacks clinical outcome evidence.
\end{abstract}

Keywords: Asthma; chronic obstructive pulmonary disease; electrical impedance tomography; flow limitation and hyperinflation

Submitted Jun 28, 2020. Accepted for publication Oct 16, 2020.

doi: 10.21037/atm-20-4984

View this article at: http://dx.doi.org/10.21037/atm-20-4984 


\section{Introduction}

Patients with obstructive lung disease (OLD) have obstructive airways due to various causes, such as inflammation, excessive mucus, and airway deformation. Because of the resulting increase in airway resistance, flow limitation and hyperinflated lung regions are common manifestations of OLD. The two main kinds of OLD are asthma and chronic obstructive pulmonary disease (COPD); these conditions are highly prevalent and cause a substantial burden on the healthcare system, patients, and their relatives (1).

OLD is generally diagnosed based on spirometry (2), which provides global information measured from the airway opening. However, as bronchial abnormalities often exhibit spatial non-uniformity that may be not assessed by spirometry, regional information may help to characterize the disease progress (3). Advanced imaging techniques (e.g., computed tomography, optical coherence tomography, and confocal laser endomicroscopy) are used to quantify structural alterations of the airways $(4,5)$. However, these imaging techniques are not suitable for longterm monitoring due to their radiation load, procedural invasiveness, and high costs. Furthermore, these methods only measure the anatomical deterioration, which is not a direct measure of lung function (6).

Electrical impedance tomography (EIT) is a functional radiation-free imaging technique that measures regional lung ventilation distribution by calculating the impedance changes in the corresponding regions (7). In recent years, the clinical use of EIT has been studied intensively (8-10), especially the use of EIT during mechanical ventilation (11), perioperative care (12), and in patients with acute respiratory distress syndrome (13). However, no review has focused on the use of EIT specifically in patients with OLD. We therefore conducted a review of the published literature on methodologies of EIT data analysis and the corresponding clinical applications in patients with flow limitation and lung hyperinflation.

We present the following article in accordance with the NARRATIVE REVIEW reporting checklist (available at http://dx.doi.org/10.21037/atm-20-4984).

\section{Methods}

The PubMed database (www.ncbi.nlm.nih.gov) was searched using the keywords "electrical impedance tomography" or "EIT" combined with "obstructive lung", "obstructive pulmonary", "obstructive airway", "obstructive sleep apnea", "asthma", "COPD”, "bronchiectasis", "bronchitis", "bronchiolitis", or "cystic fibrosis". Study titles and abstracts were screened for eligibility. Articles that were related to EIT technology and were clinically oriented were reviewed. Full texts of potentially eligible articles were retrieved. Only studies targeting patients with OLD and published as full-text articles in English journals were included. Study protocols, reviews, and editorial articles were excluded.

Data from included studies were collected using a data abstraction form. Collected data included: (I) study detailsauthor, publication year, design, objectives; (II) EIT details-purpose of use, measures, technical characteristics; (III) intervention details - validation and comparator (where applicable), intervention type (where applicable); (IV) population details—sample size, demographics; and (V) key findings.

\section{Results and discussion}

After removing duplicates, a total of 75 articles were identified. Title and abstract review led to the exclusion of 31 articles. All 44 included studies were related to OLD and EIT (Tables 1-3). Seven studies were related to simulation, animal experimentation, or reconstruction algorithm development with evaluation on patients (Table 1). Twentyseven studies were conducted to introduce and evaluate EIT-based measures, and assess the feasibility of using EIT in patients with OLD in the clinical setting (Table 2). Ten studies were designed to assess the effects of various therapies, ventilator strategies, and maneuvers (Table 3). EIT measures were used as the endpoints of the included studies.

\section{Conducting measurements}

The included studies described several measurement settings. Depending on the manufacturer of the EIT system, the number of electrodes varied from 16 to 32; for example, 16 electrodes were used with the PulmoVista 500 (Dräger Medical, Lübeck, Germany) (45), Sheffield Mark I (IBEES, Sheffield, England) (22), GeoMF II (Cardinal Health, Hoechberg, Germany) (48), KHU Mark2.5 (IIRC, Seoul, Korea) (43), Dixtal Enlight (Timpel Medical, São Paulo, Brazil) (28), and PEIT4, (FMMU, Xi'an, China) (42), while 32 electrodes were used with the Swisstom BB2 (SenTec AG, Landquart, Switzerland) (36). The electrodes 


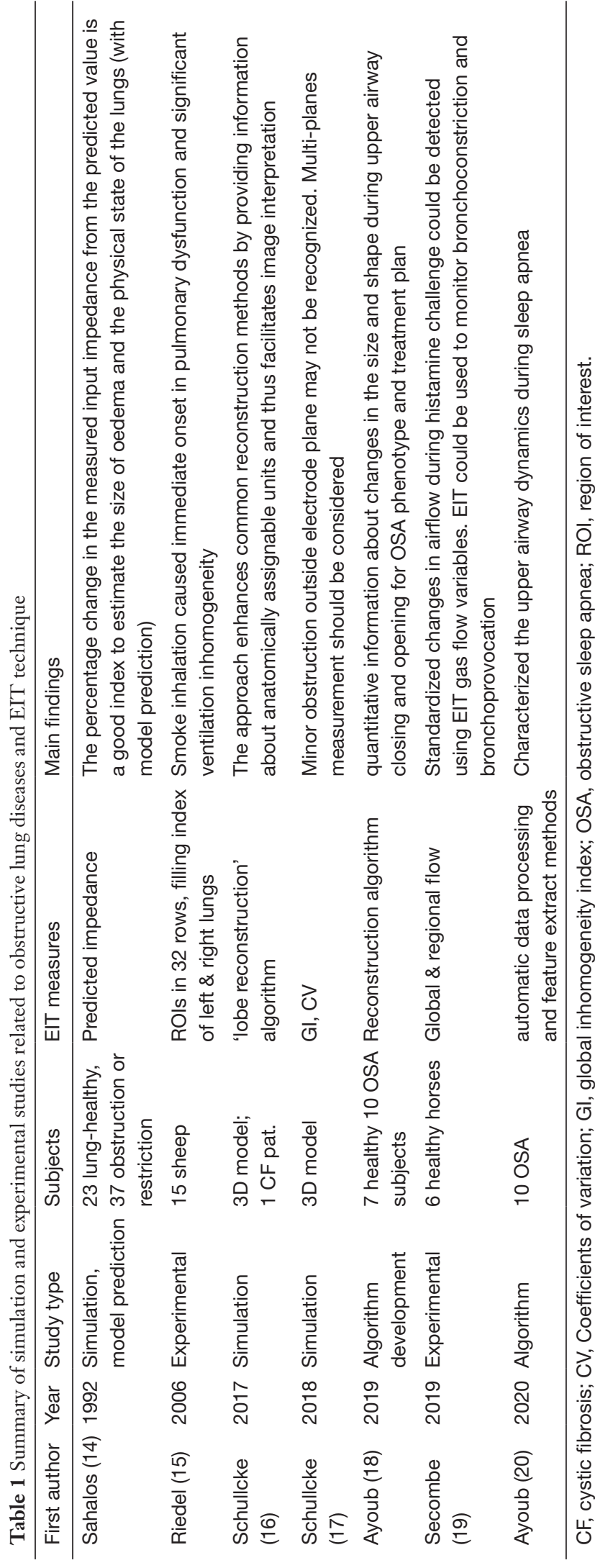

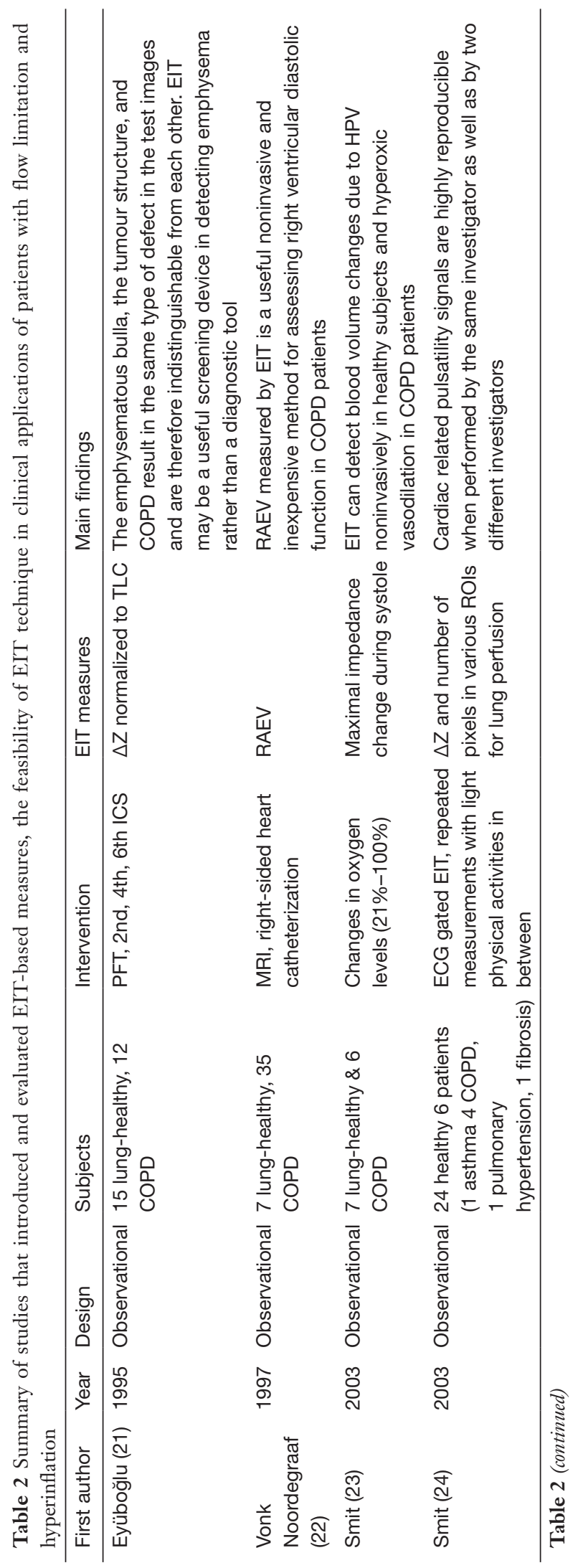




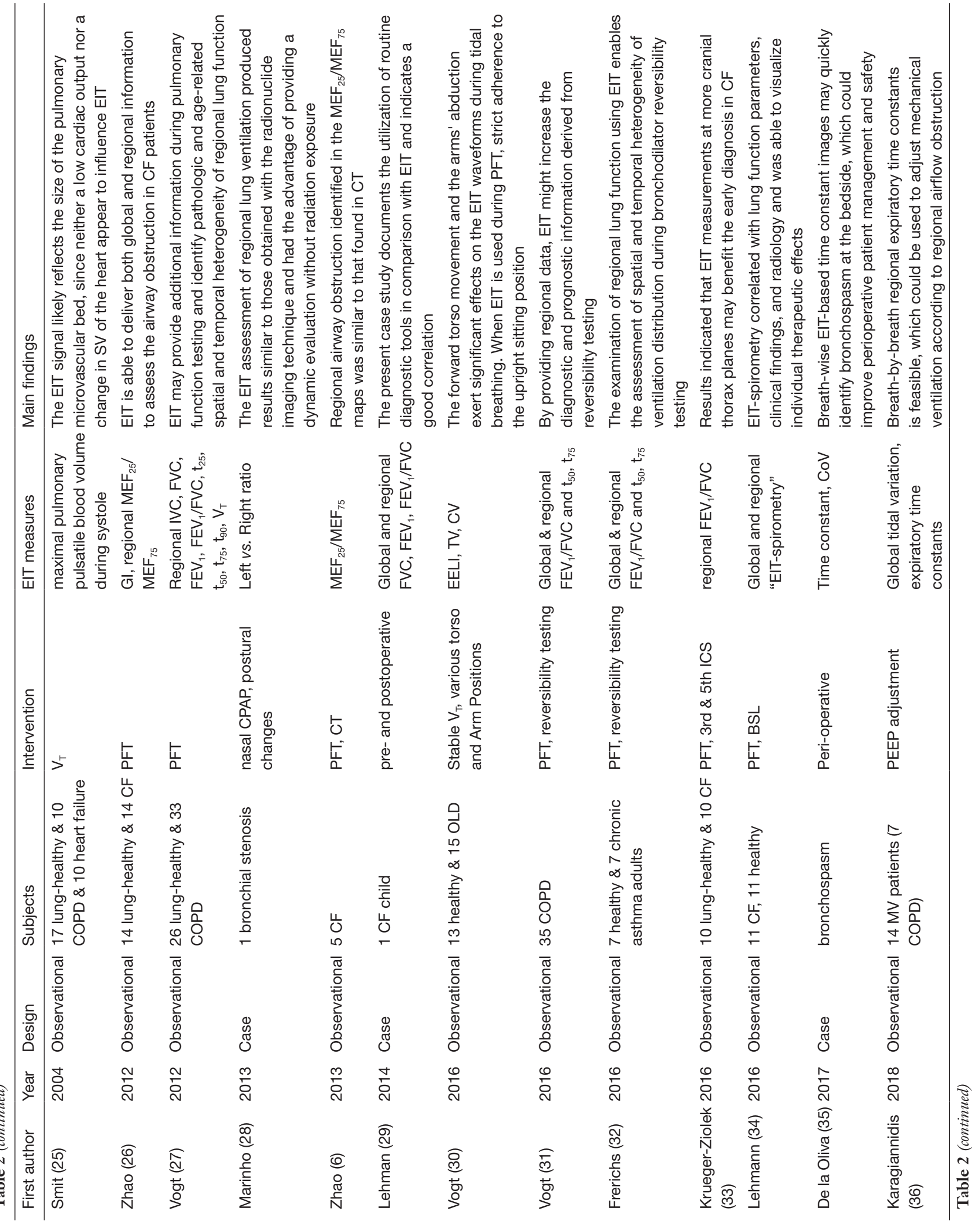




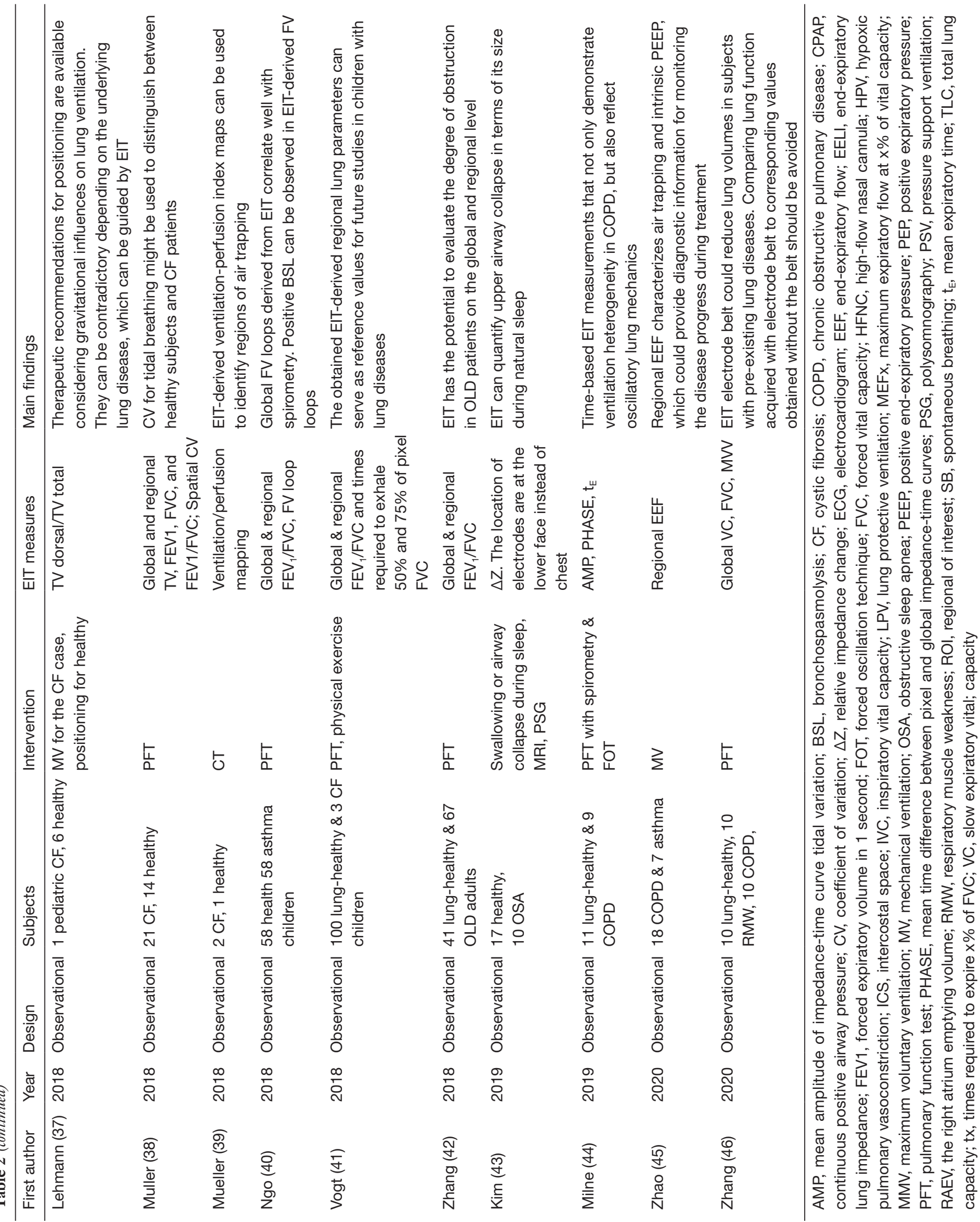




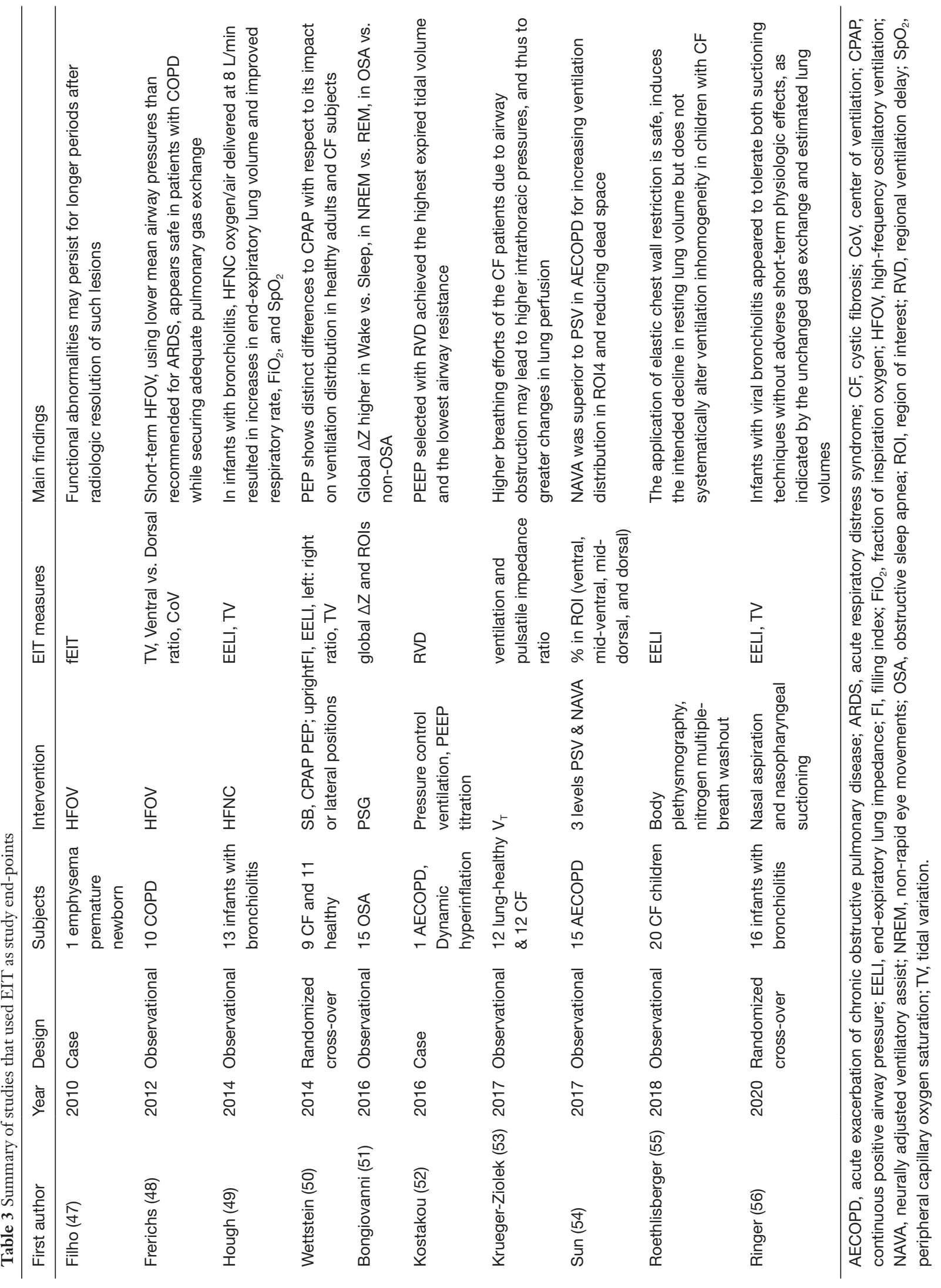



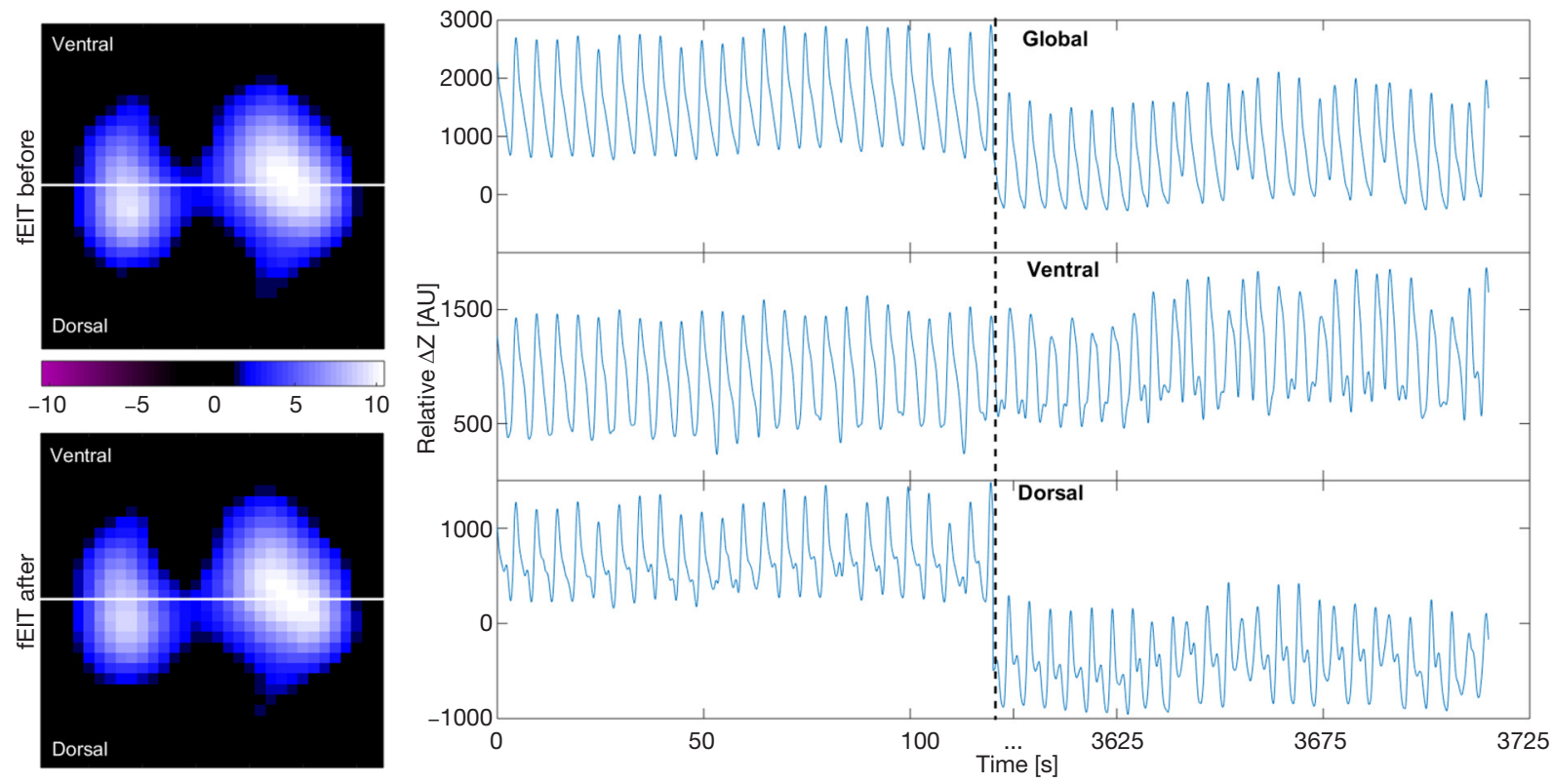

Figure 1 Functional EIT images representing tidal variation (left) and relative impedance $(\Delta \mathrm{Z})$ curves (right) from one patient with acute exacerbation of chronic obstructive pulmonary disease under assist-control ventilation. The tidal variation does not change much before versus after bronchodilator administration (left top $v s$. left bottom image). The global impedance curve shows a decrease in end-expiratory lung impedance 60 minutes post-bronchodilation (right top image), mainly caused by the changes in the dorsal regions (right bottom image) rather than the ventral regions (right middle image). EIT, electrical impedance tomography; AU, arbitrary unit.

were placed around the chest at the $4^{\text {th }}$ to $6^{\text {th }}$ intercostal spaces in most cases, except in three studies of patients with obstructive sleep apnea (OSA), where the electrodes were placed around the lower head above the neck to measure the airway occlusion $(18,20,43)$. As the impedance-volume ratio may be significantly influenced by the position of the electrode plane and volume excursion $(57,58)$, we strongly advise against the placement of electrodes lower than the $5^{\text {th }}$ intercostal space during spirometry testing. Furthermore, an adjacent current injection pattern is used for the setup of a 16-electrode system, whereas a skip-4 injection pattern is used for a 32-electrode system.

\section{EIT data evaluation}

Impedance values are presented as the relative timedifference with arbitrary units, i.e., $\Delta \mathrm{Z}=\left(\mathrm{Z}_{\mathrm{t}_{-} 1}-\mathrm{Z}_{\mathrm{t}_{-} \text {ref }}\right) / \mathrm{Z}_{\mathrm{t}_{-} \text {ref }}$. The relative impedance change $(\Delta Z)$ always refers to the impedance of a previous reference timepoint $\left(\mathrm{Z}_{\mathrm{t}_{\mathrm{t}} \mathrm{ref}}\right)$. However, normalization with $Z_{\mathrm{t}_{\text {_ref }}}$ might be skipped in some presentations. Most EIT systems were developed to monitor spatial ventilation heterogeneity; therefore, the EIT data is usually illustrated as the global impedance- time curve (sum of $\Delta Z$ from all pixels against time) and regional impedance-time curves (sum of $\Delta Z$ from regions of interest against time) (Figure 1, right images). Endexpiratory lung impedances before and after certain maneuvers that reflect the change in end-expiratory lung volume are often compared to evaluate the effects of the maneuvers $(49,55,56)$. Tidal variation (TV), which corresponds to tidal volume, is calculated by subtracting the end-expiratory global impedance from the endinspiratory global impedance. Patients with OLD typically show ventilation delay due to airway occlusion or increased mucus production, which requires extra analysis. Hence, the EIT data was evaluated offline in most included studies.

\section{Spatial ventilation distribution}

Spatial ventilation distribution is usually analyzed based on functional EIT images representing tidal ventilation (Figure 1, left images). One study discussed various types of tidal EIT (59). A direct summary of spatial ventilation distribution was achieved by calculating the global inhomogeneity (GI) or center of ventilation $(\mathrm{CoV})$ indices. The GI index quantifies the tidal ventilation distribution within the lung regions identified in tidal EIT images (60). 

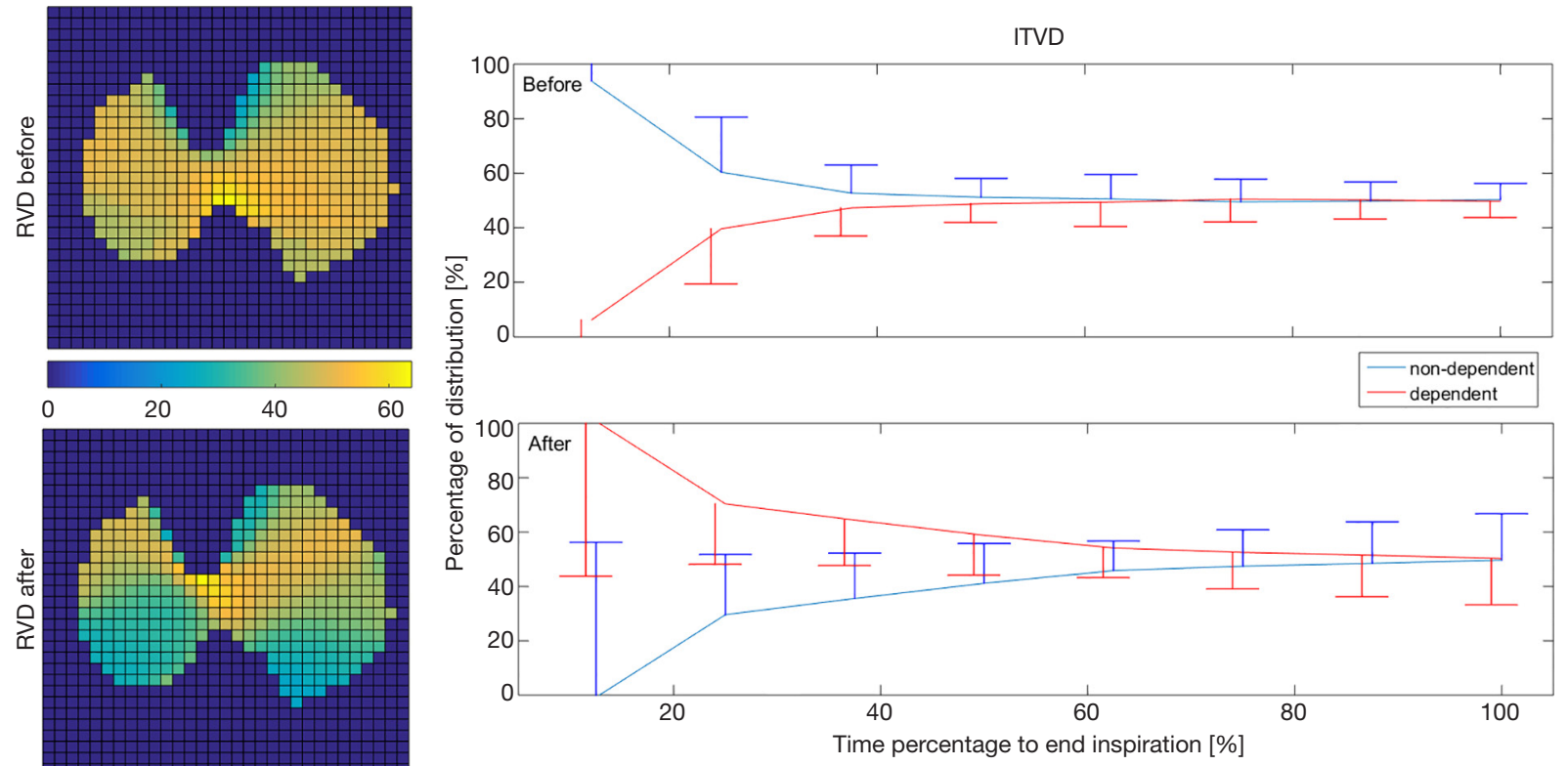

Figure 2 Regional ventilation delay (RVD; left) and intra-tidal ventilation distribution (ITVD; right) analysis of the same patient as in Figure 1. RVD maps reveal that the inspiration started soonest in the dorsal regions after bronchodilation (green regions in the left bottom image). ITVD analysis shows that the dorsal regions (gravity-dependent regions) fill faster during inspiration after bronchodilation.

The GI index is calculated from the tidal EIT images using the following equation:

$$
G I=\frac{\sum_{x, y \in \text { lung }}\left|D I_{x y}-\operatorname{Median}\left(D I_{\text {lung }}\right)\right|}{\sum_{x, y \in \text { lung }} D I_{x y}}
$$

where $D I$ denotes the value of the differential impedance in the tidal images, $D I_{x y}$ is the pixel in the identified lung area, and $D I_{\text {lung }}$ are all the pixels representing the lung area. A high GI index implies large variations among pixel tidal impedance values, indicating heterogeneous ventilation $(17,26)$. The identification of the lung area is a prerequisite for the calculation of the GI index. Any incorrect identification of the lung area omitting some sections of the lungs that are poorly or non-ventilated will decrease the apparent heterogeneity; this leads to a reduced ability of the GI index to distinguish "true" heterogeneity from the apparent effect due to the incorrect identification of lung regions.

The $\mathrm{CoV}$ characterizes the ventilation distribution in the ventrodorsal direction (61), as calculated using the following equation:

$$
\mathrm{CoV}=\Sigma\left(\mathrm{y}_{\mathrm{j}} \times \Delta \mathrm{Z}_{\mathrm{j}}\right) / \Sigma\left(\Delta \mathrm{Z}_{\mathrm{j}}\right) \times 100 \%
$$

where $\Delta Z_{j}$ is the image value in pixel $j$, and $y_{j}$ is the height of pixel $\mathrm{j}$ scaled so that the bottom of the image is $0 \%$ and the top is $100 \%$. The $\mathrm{CoV}$ is an intuitive index that compares the change in ventilation (35), especially when the tidal volume is constant. The $\mathrm{CoV}$ is sometimes simplified as the sum of the pixel TV values in the dorsal half of the image as a fraction of the global sum of the pixel TV values in the whole image (37). However, this simplification is less sensitive than the actual $\mathrm{CoV}$, and so should not be termed "CoV" (62).

\section{Temporal ventilation heterogeneity}

Obstructive airways may delay the delivery of air to the alveoli. EIT with high temporal resolution is able to capture such delays. The regional ventilation delay (RVD) quantifies the time delay needed for the regional impedance-time curve to reach a certain threshold of the maximal local impedance (63). As illustrated in Figure 2, the RVD highlights the change in the lung status after treatment. The RVD may help to identify the positive end-expiratory pressure (PEEP) level with the largest expired tidal volume and the lowest airway resistance in patients with acute exacerbation of COPD (52). Similarly, the regional time delay and regional expiratory time are correlated to the oscillatory impedance measured by the forced oscillation technique (44). During the forced vital capacity (FVC) maneuver, the time required to expire 
$\mathrm{x} \%$ of the FVC is often calculated to show the temporal heterogeneity in patients with OLD (27), and as a measure of the efficacy of bronchodilator administration $(31,32)$. The intra-tidal ventilation distribution (ITVD) shows the ventilation heterogeneity during inspiration (64). The left image in Figure 2 shows that the ITVD illustrates the temporal ventilation heterogeneity from another aspect to the RVD. Sun et al. found that the ventilation in the most dependent lung regions may be associated with diaphragm activity (54). However, while Zhao et al. evaluated the $\Delta \mathrm{Z}$ of the whole tidal breath, the division of the ITVD into the inspiration in several time periods may provide more complex information regarding diaphragm activity (65), which may be useful in the assessment of patients with spontaneous breathing. Another measure used to evaluate temporal heterogeneity is the regional time constant, which reflects changes in regional lung mechanics (resistance $x$ compliance) $(35,36)$. The impedance-time curves are fitted using the following equation:

$$
Z(t)=Z_{0} \cdot e^{-\frac{t}{\tau}}+k
$$

where $Z(t)$ denotes the impedance at timepoint $t, Z_{0}$ is the impedance at the start of the fitting period, $t$ represents the time during the fitting period, $\tau$ is the time constant, and $\mathrm{k}$ is the impedance value at the end of the fitting period. The regional time constant may be altered not only in patients with OLD, but also in patients with lung compliance changes (e.g., acute respiratory distress syndrome). The temporal heterogeneity is often presented as a histogram of the coefficient of variation and frequency distributions $(27,38)$; such presentations could also be used for other evaluation categories.

\section{Flow limitation}

The rationale of the lung EIT technique is based on the assumption of a linear relationship between $\Delta \mathrm{Z}$ and lung volume changes during inspiration and expiration, as validated in previous studies $(66,67)$. Therefore, the derivatives of $\Delta Z$ are considered to be proportional to the inspiratory and expiratory flows. Flow limitation is a wellknown symptom of OLD (2). EIT captures regional flow limitations and provides unique information that cannot be obtained using other techniques. The most widely studied parameters are obtained via EIT-based spirometry, including the forced expiratory volume in 1 second $\left(\mathrm{FEV}_{1}\right), \mathrm{FVC}$, and maximum expiratory flow at $\mathrm{x} \%$ of the vital capacity $(6,26,27,31,32,42)$. In addition, regional flow-volume loops may show inhomogeneous flow limitation among different regions of interest (40). These EIT-based spirometry parameters are calculated in a similar manner to their original definitions, except that the regional $\Delta \mathrm{Z}$ and $\Delta \mathrm{Z}^{\prime}$ are used as substitutes for the volume and flow. The regional lung function map provides an intuitive way to understand the patient's status (Figure 3). In patients with OLD under mechanical ventilation, flow limitation often prevents the end-expiratory flow from returning to zero. Regional endexpiratory flow may be associated with air trapping and intrinsic PEEP, and provide diagnostic information for monitoring the disease progress during treatment (45).

\section{Cardiac-related signals}

High temporal resolution allows EIT to study not only ventilation but also faster physiological phenomena, such as pulmonary perfusion and the pulsatility of the lung during the cardiac cycle $(68,69)$. Cardiac-related signals are highly reproducible in COPD (22-25). Vonk Noordegraaf et al. showed that the ratio of the volume change during the rapid filling phase to the total ventricular filling volume measured by EIT can be used to assess the right ventricular diastolic function (22). Recently, another group calculated the ventilation/perfusion mismatch based on cardiacrelated pulsatility changes to estimate air trapping (39). The pulsatility method measures the amplitude of the cyclic perturbations in local lung impedance caused by the passage of the stroke volume through the lung. Lung pulsatility is also significantly influenced by the distensibility of the pulmonary vessels and the size and patency of the pulmonary microvascular bed (70). Therefore, pulsatilitybased methods might be misleading as a measure of pulmonary perfusion in patients with collapse of small pulmonary vessels or substantial changes in the parenchymal architecture. Another EIT-based method using hypertonic saline bolus injection seems to be promising as a measure of lung perfusion $(69,71)$.

\section{Clinical applications}

\section{Lung function testing}

As described in the previous section, the main application of EIT for patients with flow limitation and lung hyperinflation is in the field of lung function testing (spirometry). As summarized in Tables 2 and 3, simultaneous measurement of EIT and spirometry provides not only global absolute volume and flow limitations, but also regional relative alterations caused by the disease or in response to treatment. The absolute values determined by spirometry can be used 

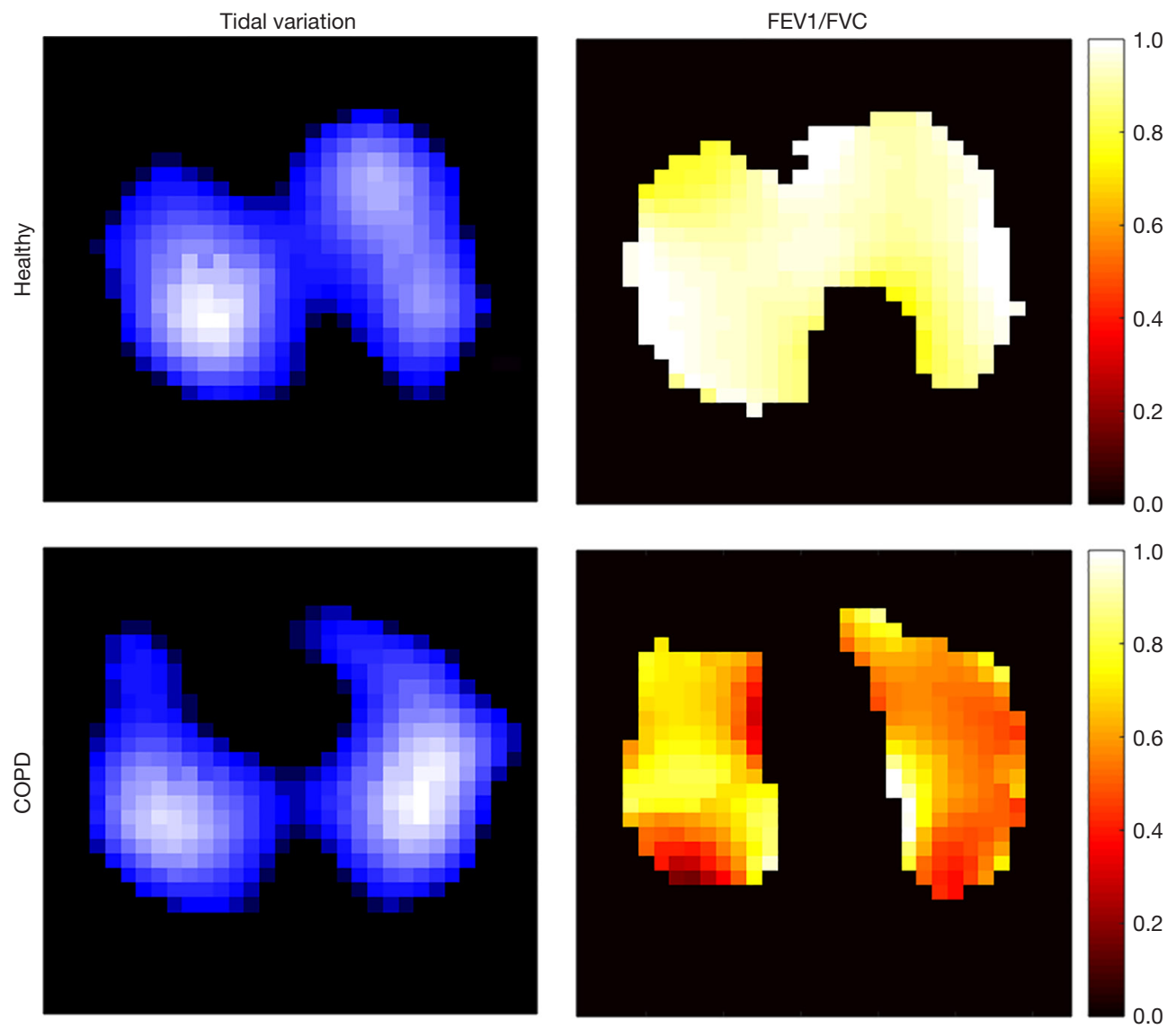

Figure 3 Functional EIT images showing the spatial ventilation distribution during tidal breathing (tidal variation; left) and the regional spirometry parameters (FEV1/FVC; right) in a healthy volunteer (top row) and a patient with chronic obstructive pulmonary disease (COPD) (bottom row). Highly ventilated regions are marked with light blue in the tidal variation maps (left). Scale is in arbitrary units. Regions with a high FEV1/FVC ratio are marked with light yellow in the EIT-based regional spirometry maps (right). Although the spatial ventilation distribution seems unaffected, the regional lung function defect is easily identifiable, especially in the left lung where the regional FEV1/ FVC is much lower. FEV1, forced expiratory volume in 1 second; FVC, forced vital capacity.

to normalize $\Delta \mathrm{Z}$ and $\Delta \mathrm{Z}^{\prime}$ to milliliters and milliliters per second. To assess the changes in functional residual capacity and residual volume, body plethysmography is used instead of spirometry. One study described the modification of a body plethysmography device to allow the simultaneous measurement of EIT and absolute lung volume to evaluate the influence of the electrode plane on EIT data (57). Such a novel combined system enables the simultaneous assessment of global and regional lung function, which may provide new possibilities in the diagnosis and prognosis of pulmonary diseases. Nevertheless, as such modification is not standard, the clinical gain versus cost needs to be evaluated in further studies. The forced oscillation technique (FOT) superimposes forced oscillation signals at the airway opening to determine the mechanical impedance of the respiratory system. The FOT is often used to examine the lung function of patients with OLD (72). A measurement modality that combines the FOT and EIT has recently been introduced (73). The correlation between EIT and FOT results suggests that such a combination is a promising future way to evaluate the respiratory system (44).

The effect of bronchodilator administration on flow limitation is usually assessed by spirometry. In the era of EIT, besides the shape of the flow-volume loop, the heterogeneity of lung function parameters in various lung regions provides deeper understanding of the drug efficacy. Patients with asthma and COPD show not only a reduction in the absolute value of flow, but also a less homogeneous 
regional lung function compared with healthy subjects $(31,32)$. Spatial and temporal ventilation distribution is improved after bronchodilator administration in patients with asthma and partially improved in patients with COPD, as evidenced by the histograms of pixel $\mathrm{FEV}_{1} / \mathrm{FVC}$ values and pixel expiration times. Further study may focus on the utility of this novel information for diagnosis and disease progress monitoring. As the forced maneuvers in lung function testing are highly dependent on the motivation and effort of the patients, future research may focus on the ability of EIT to assess regional lung function without the need for forced maneuvers. Our unpublished data from 60 patients with COPD suggests that the parameters of spatial heterogeneity obtained from quiet tidal breathing are comparable to the measures derived from the forced maneuvers.

\section{Patients receiving ventilation support}

Patients with severe COPD or asthma may require ventilation support. Several studies have shown that regional air trapping and intrinsic PEEP can be visualized with EIT $(39,45,74)$. Furthermore, two studies evaluated the use of EIT to guide PEEP adjustment in OLD $(36,52)$. Temporal information (e.g., regional delay in ventilation and expiratory time constants) can be considered to optimize PEEP to reduce flow limitation. The influences of various ventilation supports on the respiratory system have also been evaluated with EIT-based measures (47-49,54), which helps clinicians to choose the appropriate ventilation mode or type of support for patients with flow limitation and lung hyperinflation. Frerichs et al. used EIT to monitor ventilation during high-frequency oscillatory ventilation, which could not be achieved by other techniques due to the high temporal dynamics (48). They found that ventilation is more homogeneously distributed during high-frequency oscillatory ventilation than during initial conventional mechanical ventilation (48). In addition, the EIT technique is becoming more popular in infants and children, as the diagnostic and monitoring options for the respiratory system are more limited in pediatric patients compared with adults. One study showed that a high-flow nasal cannula leads to increases in end-expiratory lung volume and improvements in respiratory rate, fraction of inspiration oxygen, and peripheral capillary oxygen saturation in infants with bronchiolitis (49); in addition, suctioning techniques do not negatively affect lung volume in the same patient group (56). Furthermore, neurally adjusted ventilatory assist is superior to PSV for increasing ventilation distribution in the most dorsal regions and reducing dead space in patients with acute exacerbation of COPD (54). Pendelluft is caused by different regional time constants or dynamic pleural pressure variations in spontaneously breathing patients. We recently introduced an EIT-based method to evaluate the degree of pendelluft, thus enabling visualization of the improvement in airway obstruction after treatment (75). This information might be correlated to that derived from the time constant; this issue requires further investigation.

\section{OSA}

OSA is usually diagnosed using polysomnography in the sleep laboratory. This labor-intensive diagnosis requires overnight hospitalization, which makes in-laboratory sleep studies expensive and inconvenient. Home sleep testing is a cost-effective alternative used to diagnose moderate to severe sleep apnea; however, the reduced measurements often fail to accurately stratify the severity of the breathing disorder. EIT offers new and accurate information in addition to both polysomnography and home sleep testing by adding lung volume signals to quantify apnea and hypopnea (76). EIT around the chest wall shows that lung volume changes differ between those with versus without OSA (51). Furthermore, new electrode placement designs and reconstruction algorithms enable the detection of upper airway collapse $(18,20,43)$.

\section{Limitations}

Our study has certain limitations. The literature search may have missed pertinent articles due to the selection of the search terms. Furthermore, potentially relevant articles published in languages other than English were not included. Finally, the included studies share a common limitation of small sample size, which may limit their validity.

\section{Conclusions}

In pediatric and adult patients, EIT has been successfully validated for assessing spatial and temporal ventilation distribution, measuring changes in lung volume and flow, and studying regional respiratory mechanics. EIT has also been demonstrated to be a viable alternative or supplement for well-established measurement modalities (e.g., conventional pulmonary function test) to track the progression of OLD, although the existing literature lacks prediction values as references and lacks clinical outcome 
evidence.

\section{Acknowledgments}

Funding: This work was partially supported by the National Science and Technology Major Project (No. 2017ZX10204401), the Natural Science Foundation of Guangdong Province (2020A151501964), Everest Program of AFMU (grant no. 2019ZFB002), Science Foundation for Post Doctorate Research (2019M663988), National Natural Science Foundation of China (NSFC 51837011), BMBF MOVE (FKZ 13FH628IX6) and H2020 MCSA Rise \#872488-DCPM, Self-determined Project of GIRH (2019GIRHQ05) and Natural Science Basic Research Program of Shaanxi Province (2020JM-314). The funding sources had no involvements in study design; collection, analysis, and interpretation of data; or writing of the report.

\section{Footnote}

Reporting Checklist: The authors have completed the NARRATIVE REVIEW reporting checklist. Available at http://dx.doi.org/10.21037/atm-20-4984

Conflicts of Interest: All authors have completed the ICMJE uniform disclosure form (available at http://dx.doi. org/10.21037/atm-20-4984). Dr. ZZ reports personal fees from Draeger Medical, outside the submitted work. The other authors have no conflicts of interest to declare.

Ethical Statement: The authors are accountable for all aspects of the work in ensuring that questions related to the accuracy or integrity of any part of the work are appropriately investigated and resolved.

Open Access Statement: This is an Open Access article distributed in accordance with the Creative Commons Attribution-NonCommercial-NoDerivs 4.0 International License (CC BY-NC-ND 4.0), which permits the noncommercial replication and distribution of the article with the strict proviso that no changes or edits are made and the original work is properly cited (including links to both the formal publication through the relevant DOI and the license). See: https://creativecommons.org/licenses/by-nc-nd/4.0/.

\section{References}

1. Orts LM, Bech BH, Lauritzen T, et al. Lung function in adults and future burden of obstructive lung diseases in a long-term follow-up. NPJ Prim Care Respir Med 2020;30:10.

2. Singh D, Agusti A, Anzueto A, et al. Global Strategy for the Diagnosis, Management, and Prevention of Chronic Obstructive Lung Disease: the GOLD science committee report 2019. Eur Respir J 2019;53:1900164.

3. Gallardo Estrella L, Pompe E, Kuhnigk JM, et al. Computed tomography quantification of tracheal abnormalities in COPD and their influence on airflow limitation. Med Phys 2017;44:3594-603.

4. Goorsenberg A, Kalverda KA, Annema J, et al. Advances in Optical Coherence Tomography and Confocal Laser Endomicroscopy in Pulmonary Diseases. Respiration 2020;99:190-205.

5. Leong P, Tran A, Rangaswamy J, et al. Expiratory central airway collapse in stable COPD and during exacerbations. Respir Res 2017;18:163.

6. Zhao Z, Muller-Lisse U, Frerichs I, et al. Regional airway obstruction in cystic fibrosis determined by electrical impedance tomography in comparison with high resolution CT. Physiol Meas 2013;34:N107-14.

7. Frerichs I, Amato MB, van Kaam AH, et al. Chest electrical impedance tomography examination, data analysis, terminology, clinical use and recommendations: consensus statement of the TRanslational EIT developmeNt stuDy group. Thorax 2017;72:83-93.

8. Zhao Z, Fu F, Frerichs I. Thoracic electrical impedance tomography in Chinese hospitals: a review of clinical research and daily applications. Physiol Meas 2020;41:04TR01.

9. Putensen C, Hentze B, Muenster S, et al. Electrical Impedance Tomography for Cardio-Pulmonary Monitoring. J Clin Med 2019;8:1176.

10. Shono A, Kotani T. Clinical implication of monitoring regional ventilation using electrical impedance tomography. J Intensive Care 2019;7:4.

11. Kobylianskii J, Murray A, Brace D, et al. Electrical impedance tomography in adult patients undergoing mechanical ventilation: A systematic review. J Crit Care 2016;35:33-50.

12. Spinelli E, Mauri T, Fogagnolo A, et al. Electrical impedance tomography in perioperative medicine: careful respiratory monitoring for tailored interventions. BMC Anesthesiol 2019;19:140.

13. Bachmann MC, Morais C, Bugedo G, et al. Electrical impedance tomography in acute respiratory distress syndrome. Crit Care 2018;22:263. 
14. Sahalos JN, Vlachogiannis E, Koukourlis C, et al. Electrical impedance measurements for pulmonary disease diagnosis. Clin Phys Physiol Meas 1992;13 Suppl A:171-4.

15. Riedel T, Fraser JF, Dunster K, et al. Effect of smoke inhalation on viscoelastic properties and ventilation distribution in sheep. J Appl Physiol (1985) 2006;101:76370 .

16. Schullcke B, Gong B, Krueger-Ziolek S, et al. Lobe based image reconstruction in Electrical Impedance Tomography. Med Phys 2017;44:426-36.

17. Schullcke B, Krueger-Ziolek S, Gong B, et al. Ventilation inhomogeneity in obstructive lung diseases measured by electrical impedance tomography: a simulation study. J Clin Monit Comput 2018;32:753-61.

18. Ayoub G, Kim YE, Oh TI, et al. EIT Imaging of Upper Airway to Estimate Its Size and Shape Changes During Obstructive Sleep Apnea. Ann Biomed Eng 2019;47:9909.

19. Secombe C, Waldmann AD, Hosgood G, et al. Evaluation of histamine-provoked changes in airflow using electrical impedance tomography in horses. Equine Vet J 2020;52:556-63.

20. Ayoub G, Dang TH, Oh TI, et al. Feature Extraction of Upper Airway Dynamics during Sleep Apnea using Electrical Impedance Tomography. Sci Rep 2020;10:1637.

21. Eyuboglu BM, Oner AF, Baysal U, et al.Application of electrical impedance tomography in diagnosis of emphysema--a clinical study. Physiol Meas 1995;16:A191211.

22. Vonk Noordegraaf A, Faes TJ, Janse A, et al. Noninvasive assessment of right ventricular diastolic function by electrical impedance tomography. Chest 1997;111:12228.

23. Smit HJ, Vonk-Noordegraaf A, Marcus JT, et al. Pulmonary vascular responses to hypoxia and hyperoxia in healthy volunteers and COPD patients measured by electrical impedance tomography. Chest 2003;123:18039.

24. Smit HJ, Handoko ML, Vonk Noordegraaf A, et al. Electrical impedance tomography to measure pulmonary perfusion: is the reproducibility high enough for clinical practice? Physiol Meas 2003;24:491-9.

25. Smit HJ, Vonk Noordegraaf A, Marcus JT, et al. Determinants of pulmonary perfusion measured by electrical impedance tomography. Eur J Appl Physiol 2004;92:45-9.

26. Zhao Z, Fischer R, Frerichs I, et al. Regional ventilation in cystic fibrosis measured by electrical impedance tomography. J Cyst Fibros 2012;11:412-8.

27. Vogt B, Pulletz S, Elke G, et al. Spatial and temporal heterogeneity of regional lung ventilation determined by electrical impedance tomography during pulmonary function testing. J Appl Physiol 2012;113:1154-61.

28. Marinho LS, Sousa NP, Barros CA, et al. Assessment of regional lung ventilation by electrical impedance tomography in a patient with unilateral bronchial stenosis and a history of tuberculosis. J Bras Pneumol 2013;39:742-6.

29. Lehmann S, Tenbrock K, Schrading S, et al. Monitoring of lobectomy in cystic fibrosis with electrical impedance tomography - a new diagnostic tool. Biomed Tech (Berl) 2014;59:545-8.

30. Vogt B, Mendes L, Chouvarda I, et al. Influence of torso and arm positions on chest examinations by electrical impedance tomography. Physiol Meas 2016;37:904-21.

31. Vogt B, Zhao Z, Zabel P, et al. Regional lung response to bronchodilator reversibility testing determined by electrical impedance tomography in chronic obstructive pulmonary disease. Am J Physiol Lung Cell Mol Physiol 2016;311:L8-L19.

32. Frerichs I, Zhao Z, Becher T, et al. Regional lung function determined by electrical impedance tomography during bronchodilator reversibility testing in patients with asthma. Physiol Meas 2016;37:698-712.

33. Krueger-Ziolek S, Schullcke B, Zhao Z, et al. Multi-layer Ventilation Inhomogeneity in Cystic Fibrosis. Respir Physiol Neurobiol 2016;233:25-32.

34. Lehmann S, Leonhardt S, Ngo C, et al. Global and regional lung function in cystic fibrosis measured by electrical impedance tomography. Pediatr Pulmonol 2016;51:1191-9.

35. de la Oliva P, Waldmann AD, Bohm SH, et al. Bedside Breath-Wise Visualization of Bronchospasm by Electrical Impedance Tomography Could Improve Perioperative Patient Safety: A Case Report. A A Case Rep 2017;8:3169.

36. Karagiannidis C, Waldmann AD, Roka PL, et al. Regional expiratory time constants in severe respiratory failure estimated by electrical impedance tomography: a feasibility study. Crit Care 2018;22:221.

37. Lehmann S, Leonhardt S, Ngo C, et al. Electrical impedance tomography as possible guidance for individual positioning of patients with multiple lung injury. Clin Respir J 2018;12:68-75.

38. Muller PA, Mueller JL, Mellenthin M, et al. Evaluation 
of surrogate measures of pulmonary function derived from electrical impedance tomography data in children with cystic fibrosis. Physiol Meas 2018;39:045008.

39. Mueller JL, Muller PA, Mellenthin M, et al. Estimating regions of air trapping from electrical impedance tomography data. Physiol Meas 2018;39:05NT01.

40. Ngo C, Dippel F, Tenbrock K, et al. Flow-volume loops measured with electrical impedance tomography in pediatric patients with asthma. Pediatr Pulmonol 2018;53:636-44.

41. Vogt B, Lohr S, Zhao Z, et al. Regional lung function testing in children using electrical impedance tomography. Pediatr Pulmonol 2018;53:293-301.

42. Zhang C, Dai M, Liu W, et al. Global and regional degree of obstruction determined by electrical impedance tomography in patients with obstructive ventilatory defect. PLoS One 2018;13:e0209473.

43. Kim YE, Woo EJ, Oh TI, et al. Real-Time Identification of Upper Airway Occlusion Using Electrical Impedance Tomography. J Clin Sleep Med 2019;15:563-71.

44. Milne S, Huvanandana J, Nguyen C, et al. Timebased pulmonary features from electrical impedance tomography demonstrate ventilation heterogeneity in chronic obstructive pulmonary disease. J Appl Physiol (1985) 2019;127:1441-52.

45. Zhao Z, Chang MY, Frerichs I, et al. Regional air trapping in acute exacerbation of obstructive lung diseases measured with electrical impedance tomography: a feasibility study. Minerva Anestesiol 2020;86:172-80.

46. Zhang N, Jiang H, Zhang C, et al. The influence of electrical impedance tomography belt on lung function determined by spirometry in sitting position. Physiol Meas 2020;41:044002.

47. Filho LV, Rossi Fde S, Deutsch A, et al. Persistence of ventilatory defect after resolution of pulmonary interstitial emphysema in a preterm baby. J Matern Fetal Neonatal Med 2010;23:712-6.

48. Frerichs I, Achtzehn U, Pechmann A, et al. Highfrequency oscillatory ventilation in patients with acute exacerbation of chronic obstructive pulmonary disease. J Crit Care 2012;27:172-81.

49. Hough JL, Pham TM, Schibler A. Physiologic effect of high-flow nasal cannula in infants with bronchiolitis. Pediatr Crit Care Med 2014;15:e214-9.

50. Wettstein M, Radlinger L, Riedel T. Effect of different breathing aids on ventilation distribution in adults with cystic fibrosis. PLoS One 2014;9:e106591.

51. Bongiovanni F, Mura B, Tagliaferri C, et al. Regional distribution of ventilation in patients with obstructive sleep apnea: the role of thoracic electrical impedance tomography (EIT) monitoring. Sleep Breath 2016;20:1245-53.

52. Kostakou E, Barrett N, Camporota L. Electrical impedance tomography to determine optimal positive end-expiratory pressure in severe chronic obstructive pulmonary disease. Crit Care 2016;20:295.

53. Krueger-Ziolek S, Schullcke B, Gong B, et al. EIT based pulsatile impedance monitoring during spontaneous breathing in cystic fibrosis. Physiol Meas 2017;38:121425.

54. Sun Q, Liu L, Pan C, et al. Effects of neurally adjusted ventilatory assist on air distribution and dead space in patients with acute exacerbation of chronic obstructive pulmonary disease. Crit Care 2017;21:126.

55. Roethlisberger K, Nyilas S, Riedel T, et al. Short-Term Effects of Elastic Chest Wall Restriction on Pulmonary Function in Children with Cystic Fibrosis. Respiration 2018;96:535-42.

56. Ringer CN, Engberg RJ, Carlin KE, et al. Physiologic Effects of Nasal Aspiration and Nasopharyngeal Suctioning on Infants With Viral Bronchiolitis. Respir Care 2020;65:984-93.

57. Krueger-Ziolek S, Schullcke B, Kretschmer J, et al. Positioning of electrode plane systematically influences EIT imaging. Physiol Meas 2015;36:1109-18.

58. Karsten J, Stueber T, Voigt N, et al. Influence of different electrode belt positions on electrical impedance tomography imaging of regional ventilation: a prospective observational study. Crit Care 2016;20:3.

59. Zhao Z, Yun PJ, Kuo YL, et al. Comparison of different functional EIT approaches to quantify tidal ventilation distribution. Physiol Meas 2018;39:01NT01.

60. Zhao Z, Möller K, Steinmann D, et al. Evaluation of an electrical impedance tomography-based global inhomogeneity index for pulmonary ventilation distribution. Intensive Care Med 2009;35:1900-6.

61. Frerichs I, Hahn G, Golisch W, et al. Monitoring perioperative changes in distribution of pulmonary ventilation by functional electrical impedance tomography. Acta Anaesthesiol Scand 1998;42:721-6.

62. Frerichs I, Zhao Z, Becher T. Simple Electrical Impedance Tomography Measures for the Assessment of Ventilation Distribution. Am J Respir Crit Care Med 2020;201:386-8.

63. Wrigge H, Zinserling J, Muders T, et al. Electrical impedance tomography compared with thoracic 
computed tomography during a slow inflation maneuver in experimental models of lung injury. Crit Care Med 2008;36:903-9.

64. Lowhagen K, Lundin S, Stenqvist O. Regional intratidal gas distribution in acute lung injury and acute respiratory distress syndrome--assessed by electric impedance tomography. Minerva Anestesiol 2010;76:1024-35.

65. Zhao Z, Peng SY, Chang MY, et al. Spontaneous breathing trials after prolonged mechanical ventilation monitored by electrical impedance tomography: an observational study. Acta Anaesthesiol Scand 2017;61:1166-75.

66. Marquis F, Coulombe N, Costa R, et al. Electrical impedance tomography's correlation to lung volume is not influenced by anthropometric parameters. J Clin Monit Comput 2006;20:201-7.

67. Frerichs I, Hinz J, Herrmann P, et al. Detection of local lung air content by electrical impedance tomography compared with electron beam CT. J Appl Physiol 2002;93:660-6.

68. Frerichs I, Hinz J, Herrmann P, et al. Regional lung perfusion as determined by electrical impedance tomography in comparison with electron beam CT imaging. IEEE Trans Med Imaging 2002;21:646-52.

69. He H, Long Y, Frerichs I, et al. Detection of Acute Pulmonary Embolism by Electrical Impedance Tomography and Saline Bolus Injection. Am J Respir Crit Care Med 2020;202:881-2.

Cite this article as: Sang L, Zhao Z, Lin Z, Liu X, Zhong N, Li Y. A narrative review of electrical impedance tomography in lung diseases with flow limitation and hyperinflation: methodologies and applications. Ann Transl Med 2020;8(24):1688. doi: 10.21037/atm-20-4984
70. Schuster DP, Haller J. Regional pulmonary blood flow during acute pulmonary edema: a PET study. J Appl Physiol (1985) 1990;69:353-61.

71. Mauri T, Spinelli E, Scotti E, et al. Potential for Lung Recruitment and Ventilation-Perfusion Mismatch in Patients With the Acute Respiratory Distress Syndrome From Coronavirus Disease 2019. Crit Care Med 2020;48:1129-34.

72. Oostveen E, MacLeod D, Lorino H, et al. The forced oscillation technique in clinical practice: methodology, recommendations and future developments. Eur Respir J 2003;22:1026-41.

73. Ngo C, Spagnesi S, Munoz C, et al. Assessing regional lung mechanics by combining electrical impedance tomography and forced oscillation technique. Biomed Tech (Berl) 2018;63:673-81.

74. Mauri T, Bellani G, Salerno D, et al. Regional distribution of air trapping in chronic obstructive pulmonary disease. Am J Respir Crit Care Med 2013;188:1466-7.

75. Sang L, Zhao Z, Yun PJ, et al. Qualitative and quantitative assessment of pendelluft: a simple method based on electrical impedance tomography. Ann Transl Med 2020;8:1216.

76. Lee MH, Jang GY, Kim YE, et al. Portable multiparameter electrical impedance tomography for sleep apnea and hypoventilation monitoring: feasibility study. Physiol Meas 2018;39:124004. 